for leukotriene A4 hydrolase, which converts LTA4 to LTB4; microsomal glutathione S-transferase, which converts LTA4 to LTC4; and gamma-glutamyltransferase (LTC4 >LTD4). In contrast, Tr14, but not diclofenac strongly induced Nrf2 mRNA at 12-36 hours.

Conclusions: Tr14 and diclofenac had very different effects on the COX/LOX synthetic pathway after cutaneous wounding. Tr14 allowed normal autoinduction of COX2 mRNA by PGE2, but suppressed mRNA levels for the key enzymes in the leukotriene synthetic pathway. A likely explanation for these effects is that Tr14 strongly induced Nrf2 mRNA, which is known to co-repress the leukotriene enzymes via transcription factor Bach1.

Disclosure of Interest: None declared

DOI: 10.1136/annrheumdis-2018-eular.3779

\section{THU0022 ANALYSIS OF 47 NON-MHC ANKYLOSING SPONDYLITIS SUSCEPTIBILITY LOCI REVEALS SHARED ASSOCIATED VARIANTS ACROSS CAUCASIANS AND CHINESE HAN}

X. Zheng ${ }^{1}$, Q. Li ${ }^{1}$, X. Li ${ }^{1}$, Y. Zhang ${ }^{1}$, X. Wu ${ }^{1}$, Q. Wei ${ }^{1}$, S. Cao ${ }^{1}$, M. Yang ${ }^{1}$, Z. Lin ${ }^{1}$, Z. Liao ${ }^{1}$, J. Qi ${ }^{1}$, Q. Lv ${ }^{1}$, L. Wang ${ }^{2}$, J. Liu ${ }^{1,2}$, J. Gu$^{1}$. ${ }^{1}$ Department of Rheumatology, The Third Affiliated Hospital of Sun Yat-sen University, GuangZhou, China;

${ }^{2}$ Human Genetics, Genome Institute of Singapore, Singapore, Singapore

Background: Genetic factors play a prominent role in AS pathogenesis. So far over 40 non-MHC Ankylosing Spondylitis(AS) susceptibility loci with genomewide or suggestive significance have been initially reported in Caucasians, however, lack of association evidence of most loci was seen in Chinese Han and some results seemed controversial.

Objectives: Here, we present a systematic evaluation of 47 non-MHC AS susceptibility loci using GWAS datasets in Chinese Han.

Methods: Totally 1853 AS cases and 4048 newly matched controls in 4 cohorts were obtained, after imputation meta-analysis results of 93589 variants within 47 reported loci were extracted. Best-guess genotype data were used for interaction analysis and weighted genetic risk score model construction which was then assessed by receiver operator characteristic analysis. Functional annotation was conducted using HaploReg, RegulomeDB and rVarBase Database.

Results: We revealed 14 AS-associated variants with nominal evidence in Chinese Han, including rs10865331( $p=2.96 \mathrm{E}-9)$, rs10050860 ( $\mathrm{p}=1.84 \mathrm{E}-4)$ and rs8070463 $(p=2.81 E-4)$ and found potential associated variants within these loci. We then extracted variants in ERAP1 as well as HLA-B27 tag snp rs13202464 for HLA-B27-ERAP1 interaction analysis (figure 1). Epistatic association between ERAP1 (rs30187, rs10045403) and HLA-B27 (rs13202464) was confirmed. Among those 14 variants, rs30187 showed weaker risk effect in Chinese while rs 10050860 and rs 12504282 seemed to attribute more risk (Table 1). Genetic prediction model combining 14 variants in 11 loci with HLA-B27 achieved better discrimination ability $(\mathrm{AUC}=0.884, \quad 95 \% \mathrm{Cl}=0.873 \sim 0.895)$ than $\mathrm{HLA}-\mathrm{B} 27$ alone $(p=2.17 \mathrm{E}-6)$. We also identified some likely functional variants at these loci.

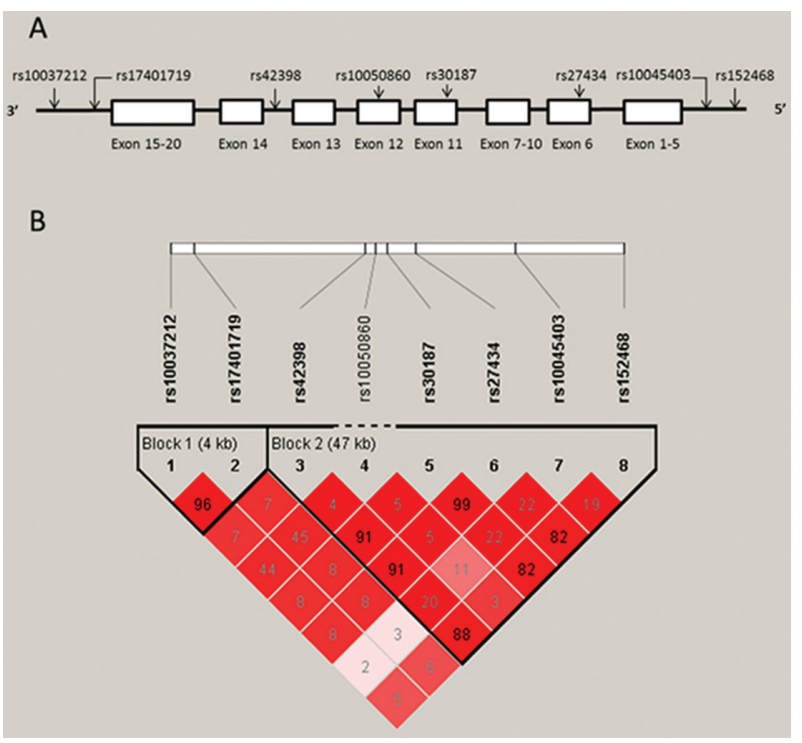

Abstract THU0022 - Figure 1
Abstract THU0022 - Table 1. Comparison of 14 shared associated non-MHC SNPs across European and Chinese

\begin{tabular}{lcccccr}
\hline Locus & SNP & $\begin{array}{c}\text { Nearby } \\
\text { Gene(s) }\end{array}$ & $\begin{array}{c}\text { Risk } \\
\text { allele }\end{array}$ & $\begin{array}{c}\text { RAF\% (CEU/ } \\
\text { CHN) }\end{array}$ & $\begin{array}{c}\text { OR } \\
\text { (CEU/CHN) }\end{array}$ & $\begin{array}{r}\text { PARP\% } \\
\text { (CEU/CHN) }\end{array}$ \\
\hline 1 p36 & rs6600247 & RUNX3 & C & $0.50 / 0.69$ & $1.16 / 1.15$ & $7.41 \% / 9.42 \%$ \\
2 p15 & rs10865331 & Intergenic & A & $0.38 / 0.49$ & $1.34 / 1.27$ & $11.44 \% / 11.60 \%$ \\
3 p24 & rs10510607 & CMC1 & C & $0.83 / 0.54$ & $1.15 / 1.14$ & $11.07 \% / 7.03 \%$ \\
$4 q 21$ & rs12504282 & ANTXR2 & T & $0.54 / 0.91$ & $1.14 / 1.20$ & $7.03 \% / 15.38 \%$ \\
5 p13 & rs11742270 & IL7R & G & $0.73 / 0.84$ & $1.11 / 1.14$ & $7.43 \% / 10.53 \%$ \\
$5 q 15$ & rs30187 & ERAP1 & T & $0.34 / 0.53$ & $1.32 / 1.11$ & $9.81 \% / 5.47 \%$ \\
$5 q 15$ & rs10045403 & ERAP1 & A & $0.73 / 0.82$ & $1.20 / 1.18$ & $12.74 \% / 12.92 \%$ \\
$5 q 15$ & rs10050860 & ERAP1 & $\mathrm{C}$ & $0.78 / 0.95$ & $1.18 / 1.45$ & $12.31 \% / 30.01 \%$ \\
$6 q 15$ & rs639575 & BACH2 & T & $0.61 / 0.49$ & $1.08 / 1.10$ & $4.65 \% / 4.69 \%$ \\
$14 q 13$ & rs8006884 & PPP2R3C & C & $0.35 / 0.40$ & $1.11 / 1.09$ & $3.71 \% / 3.44 \%$ \\
$17 q 11$ & rs2297518 & NOS2 & A & $0.19 / 0.16$ & $1.13 / 1.11$ & $2.41 \% / 1.72 \%$ \\
$17 q 21$ & rs9901869 & NPEPPS & A & $0.52 / 0.62$ & $1.15 / 1.14$ & $7.24 \% / 7.95 \%$ \\
$17 q 21$ & rs8070463 & TBKBP1 & $\mathrm{C}$ & $0.51 / 0.44$ & $1.14 / 1.16$ & $6.66 \% / 6.64 \%$ \\
$21 \mathrm{q} 22$ & rs2836883 & Intergenic & G & $0.74 / 0.83$ & $1.19 / 1.16$ & $12.33 \% / 11.71 \%$ \\
\hline
\end{tabular}

Conclusions: Our results provided a detailed spectrum of non-MHC AS susceptibility loci in Chinese Han and highlighted 2 p15, ERAP1 and TBKBP1 may play a critical role in AS pathogenesis.

\section{REFERENCES:}

[1] IGAS, Cortes A, Hadler J, et al. Nature genetics. 2013 Jul; 45(7):730-738.

[2] Ellinghaus D, Jostins L, Spain SL, et al. Nature genetics. 2016 May; 48 (5):510-518.

Disclosure of Interest: None declared

DOI: 10.1136/annrheumdis-2018-eular.6219

THURSDAY, 14 JUNE 2018:

\section{Adaptive immunity ( $T$ cells and $B$ cells) in rheumatic diseases.}

\section{THU0023 COMPLEX IMMUNOPHENOTYPING STRATIFIES PATIENTS WITH PRIMARY SJÖGREN'S SYNDROME, SYSTEMIC LUPUS ERYTHEMATOSUS AND SECONDARY SJÖGREN'S SYNDROME ASSOCIATED WITH SYSTEMIC LUPUS ERYTHEMATOSUS INTO DISTINCT CLINICALLY RELEVANT GROUPS WITH POTENTIAL THERAPEUTIC IMPLICATIONS}

N. Thompson ${ }^{1}$, A. Gandhi ${ }^{2}$, R. Radmmore ${ }^{2}$, V. Gupta ${ }^{2}$, G. Robinson ${ }^{1}$, L. Martin Gutierrez ${ }^{1}$, D. Isenberg ${ }^{2}$, E. Jury ${ }^{1}$, C. Ciurtin ${ }^{2} .{ }^{1}$ Inflammation; ${ }^{2}$ Rheumatology, University College London, London, UK

Background: Similarities in the clinical and laboratory features of patients with primary Sjögren's syndrome (pSS) and systemic lupus erythematosus (SLE) have led to attempts to treat pSS and SLE patients with similar biologic therapeutics. However, the results of many clinical trials are disappointing and no effective treatments are available for pSS and few for SLE patients with refractory disease. Objectives: To identify novel patient endotypes using in depth immune phenotyping that facilitates the selection of biological therapies for patients regardless of diagnostic labels.

Methods: Peripheral blood was collected from patients with pSS ( $n=55)$, SLE $(n=38)$, SS/SLE $(n=15)$ and age/sex-matched healthy controls $(H C s)(n=34)$. Indepth phenotyping of peripheral $\mathrm{B}$ and T-cell subsets by flow-cytometry, followed by unsupervised cluster analysis were performed. ROC analysis identified immune signatures characteristic for every cluster (endotype).

Results: Patients with pSS, SLE and SS/SLE had both unique and shared defects in immune cell phenotype. Hierarchical clustering of CD19 ${ }^{+}$B-cells, CD4 $4^{+}$ and $\mathrm{CD} 8^{+} \mathrm{T}$-cells across the three disease groups identified five distinct endotypes spanning diagnostic boundaries. Three of the endotypes had distinct immune signatures, characterised by predominantly B-cell, T-cell memory or $\mathrm{CD}^{+} / \mathrm{CD}^{+} \mathrm{T}$-cell subset fingerprints respectively, while two clusters had no distinct immune profiles. Notably, clinical and disease features were not significantly different between clusters. 

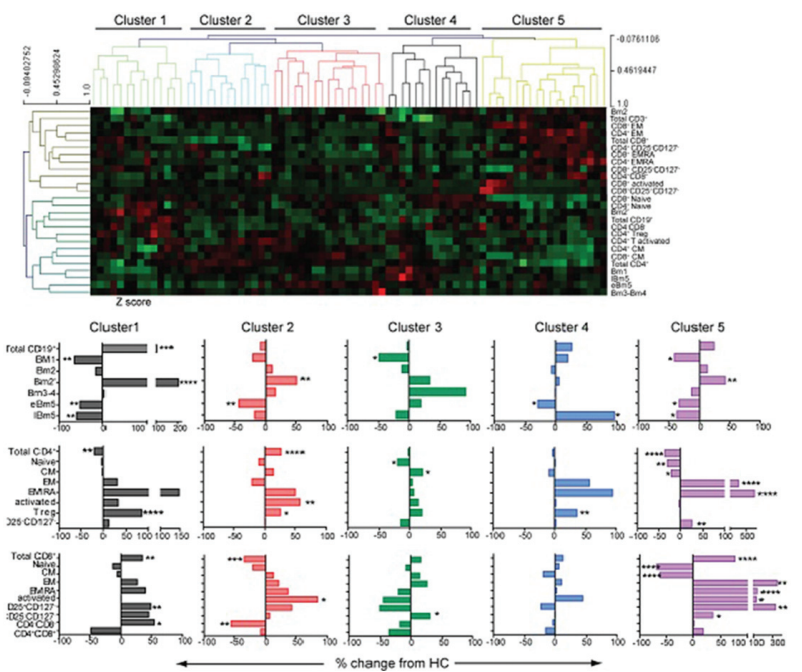

Conclusions: The results demonstrated that immune cell biomarkers could be used to re-classify patients in a manner that reflects their underlying immunopathogenesis. Characterisation of a patient's endotype could lead to better stratification of patients for selection of therapeutic targets in clinical trials.

\section{REFERENCES:}

[1] Nocturne, G. \& Mariette, X. Advances in understanding the pathogenesis of primary Sjogren's syndrome. Nat Rev Rheumatol 2013;9:544-556.

[2] Sada, P.R., Isenberg, D. \& Ciurtin, C. Biologic treatment in Sjogren's syndrome. Rheumatology (Oxford) 2015;54:219-230.

Disclosure of Interest: None declared

DOI: 10.1136/annrheumdis-2018-eular.2696

\section{THU0024 TREATMENT WITH IMMUNE CHECKPOINT INHIBITORS AND THE BREAK OF B-CELL TOLERANCE TO AUTOANTIGENS}

E.C. de Moel ${ }^{1}$, L. Rozeman ${ }^{2}$, A. Grummels ${ }^{3}$, E.M.E. Verdegaal ${ }^{4}$, J.A. Bakker ${ }^{3}$, E. H. Kapiteijn ${ }^{4}$, T.W.J. Huizinga ${ }^{1}$, J.B. Haanen ${ }^{2}$, R.E.M. Toes ${ }^{1}$, D. van der Woude ${ }^{1}$. ${ }^{1}$ Rheumatology, Leiden University Medical Center, Leiden; ${ }^{2}$ Medical Oncology and Immunology, Netherlands Cancer Institute, Amsterdam; ${ }^{3}$ Clinical Chemistry and Laboratory Medicine; ${ }^{4}$ Medical Oncology, Leiden University Medical Center, Leiden, Netherlands

Background: The field of autoimmunity may benefit from the knowledge gained by studying immune checkpoint inhibitors. These agents, which have proven remarkably successful in treating various types of cancer, inhibit negative costimulatory signals to T-cells, thereby enhancing anti-tumour T-cell responses. This can come at the cost of severe immune-related adverse effects (irAEs) including arthritis, colitis, endocrine diseases, hepatitis, and various skin abnormalities. However, it is currently unknown to which extent or to which autoimmune diseaseassociated autoantigens autoantibodies are formed (as a reflection of breaking of tolerance to self-antigens) under checkpoint inhibitor therapy and whether this is associated with irAEs.

Objectives: To investigate whether patients treated with immune checkpoint inhibitors develop autoantibodies, and whether this trait is associated with irAEs.

Methods: In pre- and (12 weeks) post-treatment sera of 133 patients with Stage III or IV melanoma treated with ipilimumab (anti-CTLA-4), we determined antibodies associated with rheumatoid arthritis (RF IgM, anti-CCP2), autoimmune hepatitis (anti-smooth muscle, anti-mitochondria, anti-liver-kidney-microsome), thyroiditis (anti-thyroid peroxidase (TPO), anti-thyroglobulin (TG)), Coeliac's disease (anti-endomysium, anti-gliadine lgG), adrenal insufficiency (anti-adrenal cortex), and autoimmune connective tissue diseases (anti-nuclear antibodies, antidsDNA, anti-ENA, and specific ENA tests: anti-SSA, anti-SSB, anti-RNP70, antiU1RNP, anti-Sm, anti-Jo1, anti-CENP, anti-PMSCL, anti-RNA polymerase 3, anti-Scl70). We used McNemar's exact test for paired data to test whether autoantibody positivity increased post-treatment, and investigated by Fisher's exact tests whether developing autoantibodies was associated with system-specific (Grade 3 or 4) irAEs.

Results: In total, post-treatment positivity for any autoantibody was seen in $19.2 \%(19 / 99)$ of patients that were fully autoantibody-negative pre-treatment $(p<0.0001)$. A significant association was observed between development of any autoantibodies and any irAEs: $14 / 19$ (73.7\%) patients that developed autoantibodies had irAEs, versus $37 / 80(46.3 \%)$ patients that did not develop autoantibodies (OR: 3.3 [95\% Cl: 1.1 to 9.9]). Regarding specific autoantibodies, predominantly anti-TPO $(4.8 \%, 6 / 125)$ and anti-TG antibodies $(6.0 \%, 8 / 132)$ developed in patients negative for these autoantibodies at baseline $(p=0.03$ and $p=0.008$, respectively). However, development of these antibodies was not associated with development of thyroid disease. For most other autoantibodies, including RA-associated antibodies, post-treatment positivity increased only marginally and was not associated with occurrence of irAEs in the organ system related to the specificity of the autoantibody.

Conclusions: Breaking of humoral tolerance as measured by development of autoantibodies is relatively common under treatment with ipilimumab and is associated with the development of irAEs. The nature of the autoantigens towards which tolerance is broken is not reflected in the phenotype of the irAEs.

Disclosure of Interest: None declared

DOI: 10.1136/annrheumdis-2018-eular.3017

\section{THU0025 \\ FLORID SYNOVITIS AFTER PD1 ANTAGONIST THERAPY IS CHARACTERISED BY A MARKED ABSENCE OF PD1+ INFILTRATING T CELLS}

W. Murray-Brown ${ }^{1}$, T. Wilsdon ${ }^{2}$, H. Weedon ${ }^{1}$, S. Proudman ${ }^{3}$, M.D. Smith ${ }^{1}$, J. Walker ${ }^{2}$, M.D. Wechalekar ${ }^{1,2},{ }^{1}$ Rheumatology, Flinders University, ${ }^{2}$ Flinders Medical Centre; ${ }^{3}$ Royal Adelaide Hospital, Adelaide, Australia

Background: Although immunological blockade of checkpoint inhibitors (Cls) for cancer therapy is known to be associated with exacerbated inflammation recapitulating many features of autoimmunity ${ }^{1}$, including synovitis resembling rheumatoid arthritis (RA $)^{2}$, no reports have investigated cellular infiltrates in synovial tissue (ST) of these patients. Here we provide the first report on ST cell infiltration, in particular PD1 expressing T cells, after a PD1 inhibitor-induced (Nivolumab) immune related adverse event (irAE) and severe synovitis.

Objectives: To characterise ST cellular infiltration in PD1 inhibitor induced arthritis with particular reference to PD1 positive T cells and compare these changes with active early RA ST.

Methods: Arthroscopic ST biopsies, parallel synovial fluid (SF) and PBMCs were collected from a DMARD-naïve nivolumab-treated small cell lung cancer (SCLC) patient with severe peripheral inflammatory polyarthritis (negative RF and ACPA no axial or extra-articular irAE); 3 DMARD-naïve patients with seropositive early RA ( $<12$ months duration; fulfilling 2010 ACR/EULAR criteria) were used as comparators.

Serial sections from fresh-frozen ST blocks were stained with $\mathrm{H}$ and $\mathrm{E}, \mathrm{CD} 3$, CD45RO, CD55 and CD68 and semi-quantitatively scored as described ${ }^{3}$. ST, SF and PBMC cell suspensions were stained with Zombie UV (BioLegend), CD45RO, PD1, CD3, ICOS, CD8, CD4, CD20 (all BD) prior to flow cytometry. Cells were gated on live, singlet, lymphocytes, CD3+ and CD4+ T cells, and CD20+ and CD8+ T cells were excluded from endpoint PD1+, ICOS+ and CD45RO+analysis.

Results: CD68 +macrophage, CD20+ B cell and CD3+ T cell and CD45RO +memory T cell infiltration in IC-irAE was comparable to RA ST on semi-quantitative scoring, while TNF; staining was markedly elevated in Cl-irAE compared to RA (Cl-irAE-TNF; 4, RA-TNF; 2). Flow cytometry identified a striking absence of PD1+ ICOS+ CD4+T cells in IC-irAE SCLC in all compartments (Cl-irAE: ST; 0.06, SF; 0.01, PBMCs; 0.00 ) compared to RA (RA: ST mean and SEM; 22.13 \pm 3.63 : SF; $45.95 \pm 1.85$ : and to a lesser extent in PBMCs; $0.41 \pm 0.13$ : $n=3$ for each), despite comparable CD4 $+\mathrm{T}$ cell frequency in each compartment (frequency of CD3 +cells, Cl-irAE: ST; 57.8, SF; 64.7, PBMCs; 38.2, RA: ST; 45.9 \pm 15.3 , SF; 49.5 \pm 11.2 , PBMCs; $62.2 \pm 13.8$, figure 1).

PD-1+ICOS+ cell frequency



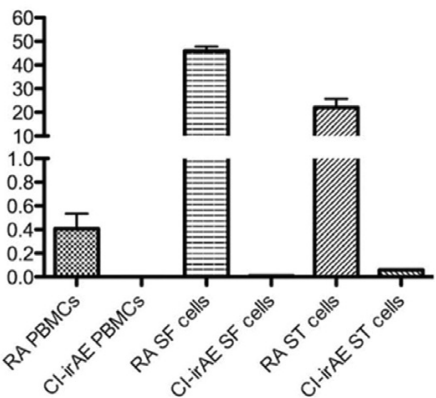

Figure 1 PD-1 + ICOS + T cells are absent in Cl-irAE. Showing the PD$1+I C O S+$ frequency of CD4 $+T$ cells gated on live, singlet, lymphocytes, and 